Supporting Information For:

\title{
Molecular Adsorption and Transport at Liposome Surfaces Studied by Molecular Dynamics Simulations and Second Harmonic Generation Spectroscopy
}

Prakash Hamal, Huy Nguyenhuu, Visal Subasinghege Don, Raju R. Kumal, Revati Kumar, Robin L. McCarley, and Louis H. Haber*

Department of Chemistry, Louisiana State University, Baton Rouge, Louisiana 70803-1804, United States

\section{Lipid Concentration Determination and Liposome Size Distributions}

The total lipid concentrations were determined by a modified Bartlett assay, which is based on the colorimetric determination of inorganic phosphate, and has been reported previously. ${ }^{1}$ Briefly, phospholipids in liposomes were converted to inorganic phosphate by perchloric acid, followed by the formation of phosphor-molybdic acid after the addition of ammonium molybdate in an acidic condition. The phosphor-molybdic acid was then reduced by Fiske-Subbarow reducer, producing a blue solution, and its absorbance was measured at $800 \mathrm{~nm}$. The calibration curve for the Bartlett assay, as shown in Figure S1, is fit with a linear equation with a slope of $0.00326 \pm$ 0.00004 and a $y$-intercept of $-0.002 \pm 0.003$. The $\mathrm{R}^{2}$ value for the fit is 0.9991 . The zeta potentials of different liposomes at $5 \mathrm{mM}$ citrate buffer of $\mathrm{pH} 4.0$ is displayed in Table S1. Similarly, the representative DLS size distributions are shown in Fig S2. The representative sizes obtained are $137 \pm 42 \mathrm{~nm}, 156 \pm 58 \mathrm{~nm}, 151 \pm 64 \mathrm{~nm}$, and $158 \pm 67 \mathrm{~nm}$ respectively for DOPG, DOPS, DOPC, and QPADOPE liposomes. Similarly, the polydispersity index (PDI) values are 0.07, 0.09, 0.10, and 0.20 respectively for DOPG, DOPS, DOPC, and QPADOPE liposomes. Zeta potential and dynamic light scattering measurements are taken using a Zetasizer Nano ZS from Malvern 
Instruments Inc., UK. Absorption measurements are done using a UV-vis spectrometer from PerkinElmer, Boston, MA, U.S.A.

Table S1. Zeta Potentials of Different Liposome Samples

\begin{tabular}{|c|c|c|c|c|}
\hline \multirow{2}{*}{$\begin{array}{c}\text { Buffer } \\
(\mathrm{pH} \mathrm{4.0)}\end{array}$} & \multicolumn{4}{|c|}{ Zeta Potential (mV) } \\
\cline { 2 - 5 } & DOPG & DOPS & QPADOPE & DOPC \\
\hline $\begin{array}{c}5.0 \mathrm{mM} \\
\text { citrate }\end{array}$ & $-73.2 \pm 1.1$ & $-74.8 \pm 1.7$ & $-67.3 \pm 1.5$ & $-21.7 \pm 2.3$ \\
\hline
\end{tabular}

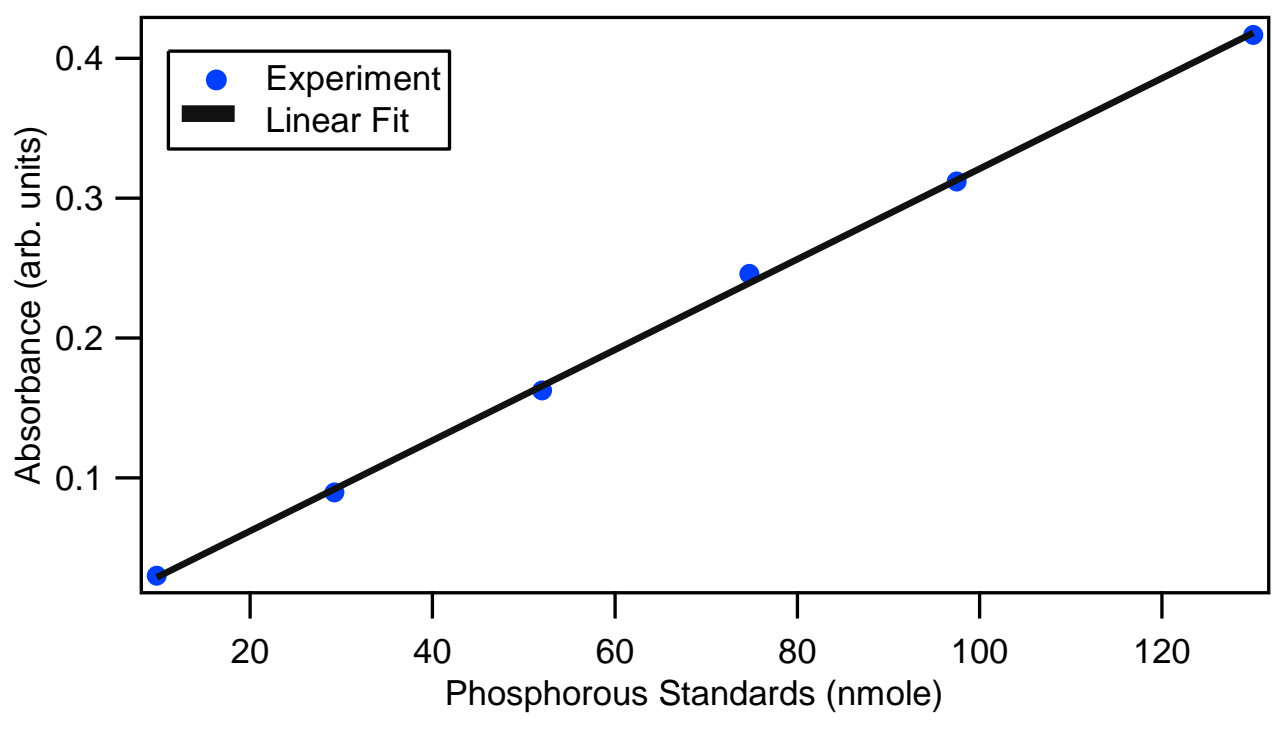

Figure S1: Bartlett assay calibration for determining the lipid concentration. 


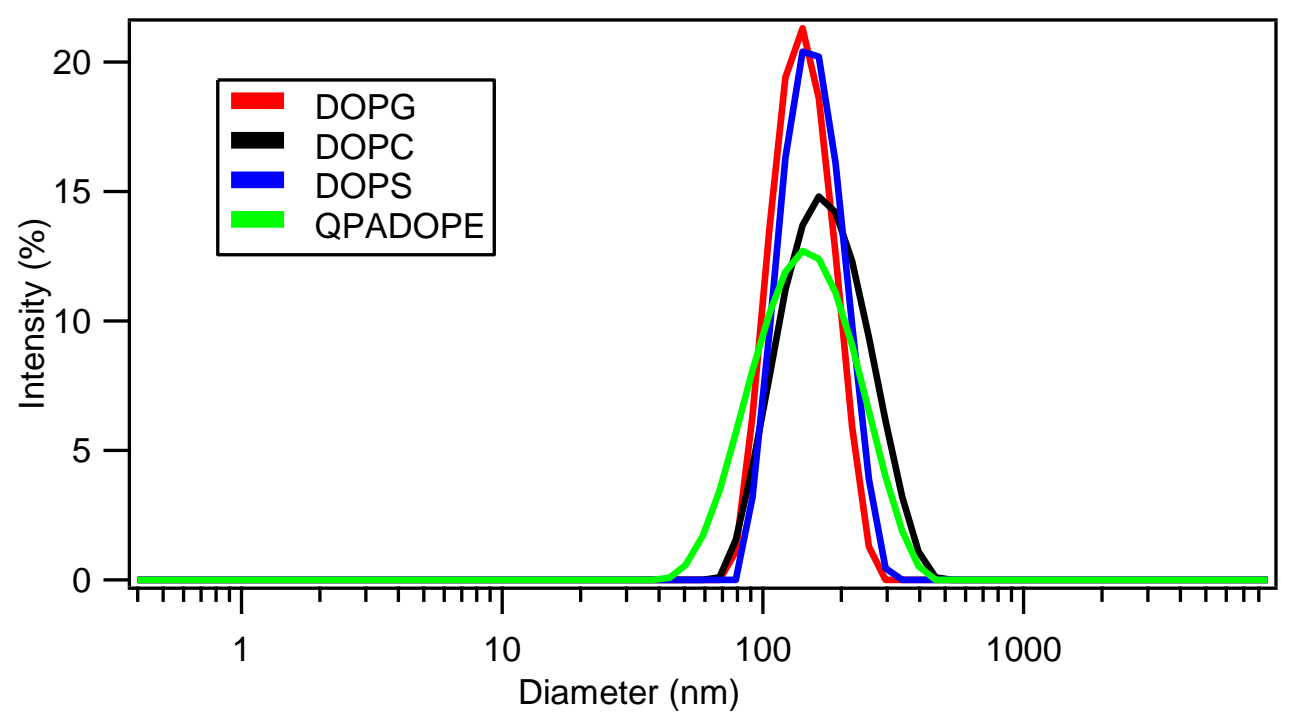

Figure S2: Size distributions measured by dynamic light scattering for DOPG, DOPC, DOPS, and QPADOPE liposomes.

\section{SHG Adsorption Isotherm to PSS Microparticles}

Adsorption isotherms for malachite green isothiocyanate (MGITC) adsorbing to the surface of polystyrene sulfate (PSS) microspheres in aqueous colloidal suspension are determined using SHG measurements for comparison to the liposome results presented in the manuscript. The PSS microspheres are purchased from Polysciences and are diluted in nanopure water to a concentration of $1.8 \times 10^{10}$ particles $/ \mathrm{mL}$. The experimental setup is the same as the one used for the liposome measurements, with the same data analysis procedure. ${ }^{1,2}$ To our knowledge, no work has previously reported SHG measurements for MGITC adsorbing to polystyrene microspheres in water. MGITC shows an SHG signal that is approximately 12 times greater than from MG at the same concentration of $0.75 \mu \mathrm{M}$, as shown in Fig S3a. The experimentally obtained isotherms are fit with the modified Langmuir isotherm model as displayed in Fig S3b. Using the modified Langmuir model, the corresponding adsorption free energy $\Delta G$ is $-13.6 \pm 0.05 \mathrm{kcal} / \mathrm{mol}$ with an 
equilibrium constant $K$ value of $(9.9 \pm 0.9) \times 10^{9}$ and $N_{\max }$ value of $0.2 \pm 0.003 \mu \mathrm{M}$. The adsorption free energy of MGITC in PSS is stronger in comparison to liposomes and is in general agreement with the literature trends. ${ }^{4}$ Similarly, the adsorption free energy also correlates that the electrostatic binding of MGITC in polystyrene sulfate microspheres is stronger than that of malachite green (MG). The reported value for the adsorption free energy of MG in polystyrene sulfate microspheres is $-11.1 \pm 0.1 .^{2,3}$ Interestingly, the reported $N_{\max }$ value of MG in polystyrene sulfate microspheres is $1.2 \pm 0.2 \mu \mathrm{M}^{3}$, which is approximately 6 times larger than the MGITC. ${ }^{1,2}$ A similar trend has been reported in literature where a strong electrostatic attraction between triphenylmethane dye molecules and colloidal polystyrene microspheres leads to higher dye-dye repulsions, leading to diminished values of $N_{\max } .^{2}$ 

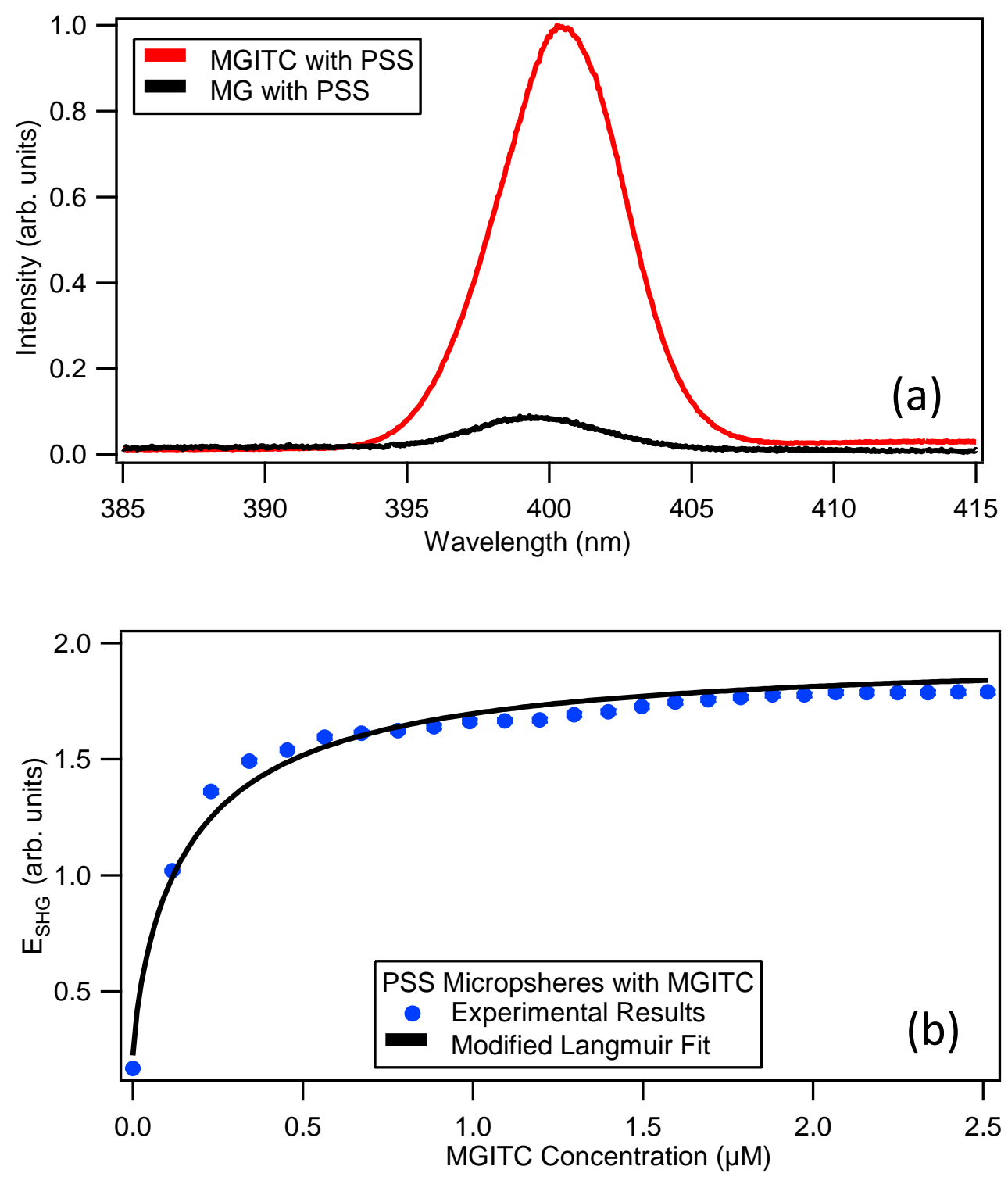

Figure S3. (a) Representative SHG spectra of polystyrene sulfate microspheres with $0.75 \mu \mathrm{M}$ of MGITC and MG. (b) SHG-determined adsorption isotherm of MGITC with polystyrene sulfate microspheres. 


\section{Additional Details on SHG Measurements of Molecular Interactions with Liposomes}

Figure $\mathrm{S} 4 \mathrm{a}$ shows the extinction spectrum of MGITC in citrate buffer at $\mathrm{pH} 4.0$ for different experimental times indicating the stability of the MGITC dye in solutions under these conditions. Additionally, a schematic energy level diagram of the MGITC dye is shown in Figure S4b, where resonance enhancement at $2 \omega$ occurs corresponding to the transition from ground state $\mathrm{S}_{0}$ to the vibrationally excited $S_{2}$ state. The transport times $\tau$ of MGITC through the bilayers of DOPG, DOPS and DOPC liposomes are listed in Table S2. Similarly, the obtained rate constants $\left(\tau^{-1}\right)$ for liposomes also have a similar trend as the transport times, as shown in Figure S5. The interaction of MGITC with the QPADOPE liposomes and with $5.0 \mathrm{mM}$ citrate buffer of $\mathrm{pH} 4.0$ is also studied using SHG measurements, as displayed in Figure S6a and S6b. These corresponding SHG intensities are plotted as a function of concentration with linear fits, as shown in Figure S7a and S7b. These SHG signal intensities are normalized consistently with Figures 2 and 3 in the

manuscript as well as Figures S6a and S6b such that $I_{S H G}=E_{S H G}^{2}$. The obtained slopes from the fits are $(0.017 \pm 0.001)$ and $(0.015 \pm 0.001) \mu \mathrm{M}^{-1}$ for Figures $\mathrm{S} 7 \mathrm{a}$ and $\mathrm{S} 7 \mathrm{~b}$, respectively. These corresponding SHG intensities are approximately equal indicating no observed adsorption to the QPADOPE liposomes, to within experimental uncertainty. Additionally, the SHG time traces in Figure S6a do not change with time, confirming that no transport occurs. As described in the manuscript, these signals are understood to be primarily from hyper Rayleigh scattering from MGITC. The value of the slope from Figure S7b is used for $\alpha$ in the SHG fits described in Equation 2 of the manuscript.

In order to investigate the possible counterion effect, the transport kinetics of the MGITC dye molecule are studied with DOPG liposomes in $5.0 \mathrm{mM}$ citrate buffer of $\mathrm{pH} 4.0$ with no additional potassium perchlorate and with $5.0 \mathrm{mM}$ additional potassium perchlorate (PP) at two 
different MGITC concentrations. The SHG time traces, shown in Figure S8a only shift down slightly upon addition of PP, similar to our previous studies with MG and potassium chloride $(\mathrm{KCl}) .{ }^{1}$ These results indicate that the perchlorate ion does not cause significant or unexpected counterion effects with MGITC. Additionally, there is no significant change in the MGITC spectrum upon addition of $5.0 \mathrm{mM} \mathrm{PP}$, and the dye remains stable over time, as shown in the extinction spectra in Figure S8b.
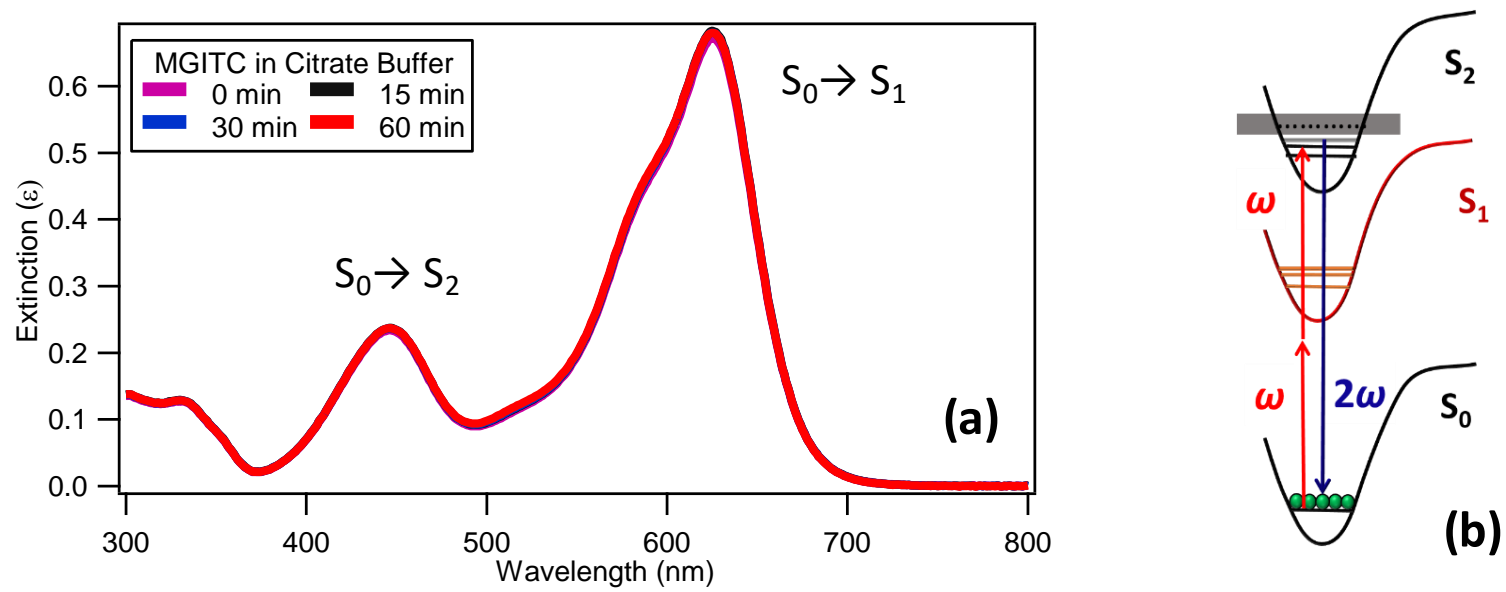

Figure S4. (a) Extinction spectrum of MGITC in citrate buffer of $\mathrm{pH} 4.0$ at various times. (b) Energy level diagram of MGITC dye molecule, showing resonance enhancement of SHG.

Table S2: Transport times of MGITC in DOPC, DOPS and DOPG liposomes

\begin{tabular}{|c|c|c|c|}
\hline \multirow{2}{*}{ MGITC $(\mu \mathrm{M})$} & \multicolumn{3}{|c|}{ Transport Time (second) } \\
\cline { 2 - 4 } & DOPC & DOPS & DOPG \\
\hline 0.16 & $500 \pm 81$ & $364 \pm 32$ & $360 \pm 17$ \\
\hline 0.53 & $400 \pm 40$ & $338 \pm 21$ & $327 \pm 12$ \\
\hline 1.06 & $334 \pm 25$ & $280 \pm 20$ & $304 \pm 6$ \\
\hline 1.31 & $238 \pm 16$ & $114 \pm 4$ & $168 \pm 10$ \\
\hline 1.57 & $222 \pm 17$ & $158 \pm 5$ & $156 \pm 9$ \\
\hline 1.94 & $208 \pm 17$ & $121 \pm 6$ & $123 \pm 8$ \\
\hline 2.06 & $200 \pm 8$ & $143 \pm 9$ & $115 \pm 12$ \\
\hline 2.55 & $167 \pm 5$ & $133 \pm 5$ & $83 \pm 4$ \\
\hline 3.44 & $143 \pm 4$ & $121 \pm 3$ & $67 \pm 3$ \\
\hline 4.75 & $125 \pm 3$ & $130 \pm 6$ & $55 \pm 3$ \\
\hline
\end{tabular}



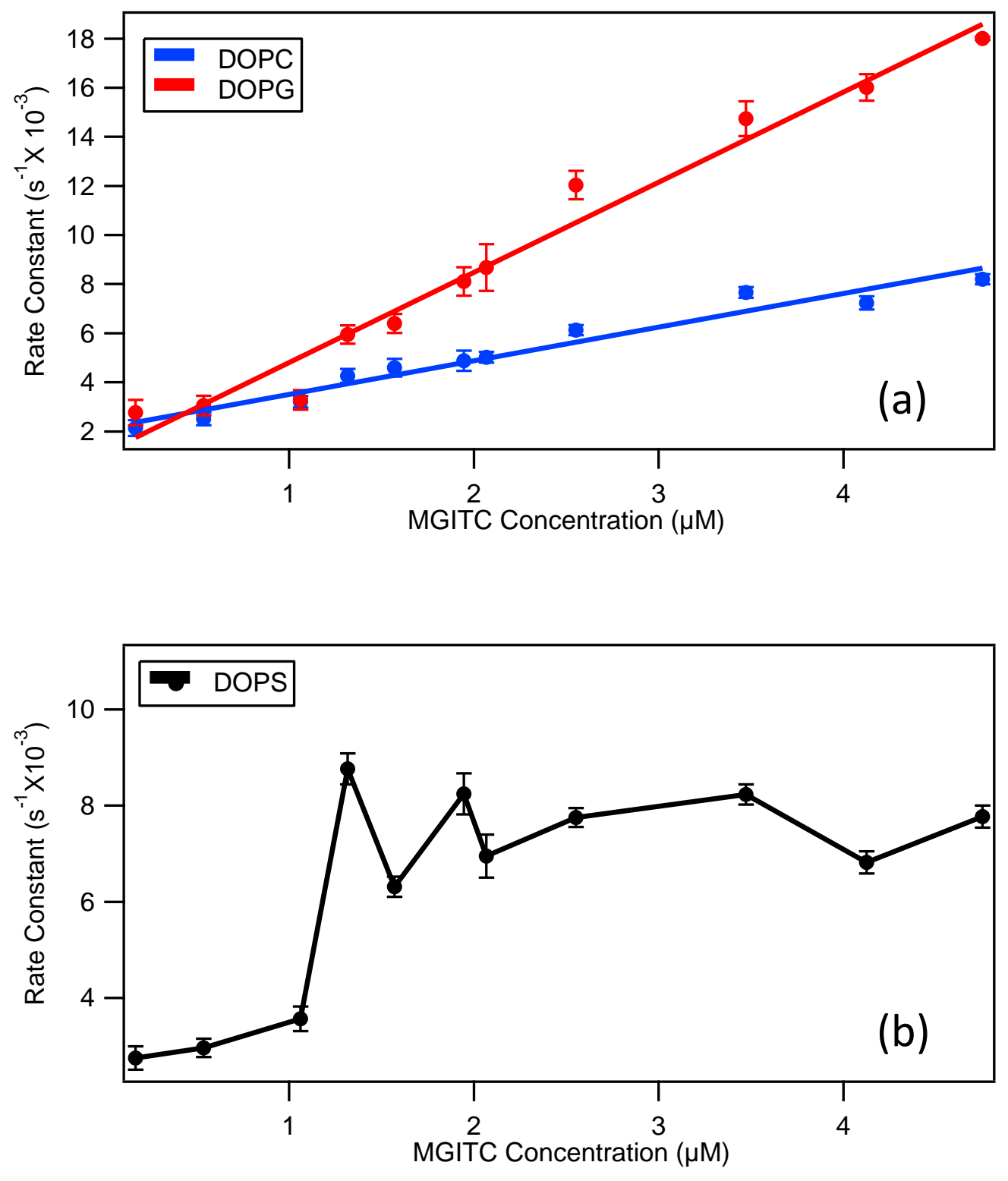

Figure S5. Variation of the transport rate constant as a function of MGITC concentration for (a) DOPG, DOPC, and (b) DOPS liposomes in $5 \mathrm{mM}$ citrate buffer of $\mathrm{pH} 4.0$. 

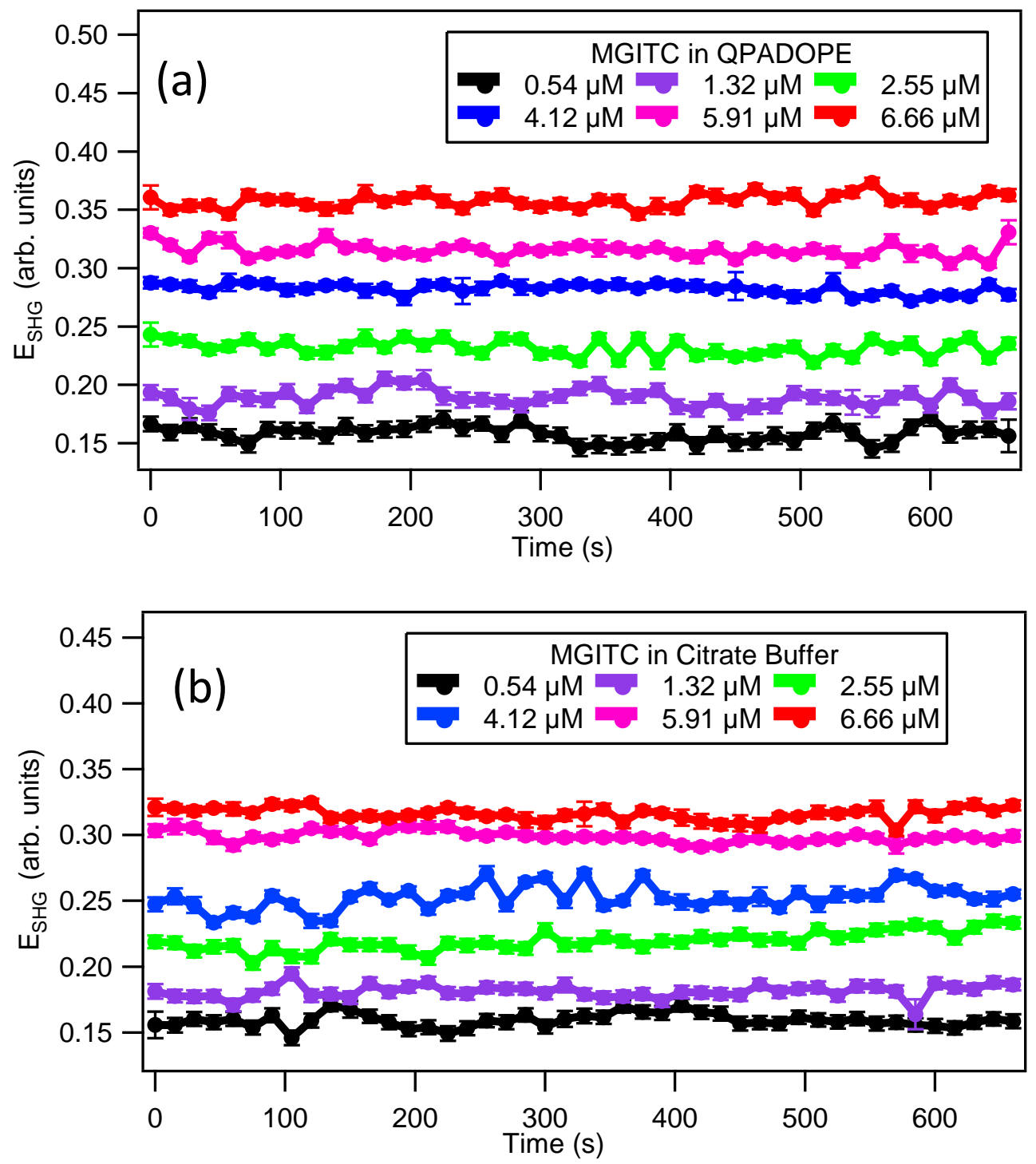

Figure S6. SHG time profiles upon addition of various concentrations of MGITC to (a) QPADOPE liposomes and (b) $5 \mathrm{mM}$ citrate buffer at $\mathrm{pH} 4.0$. 

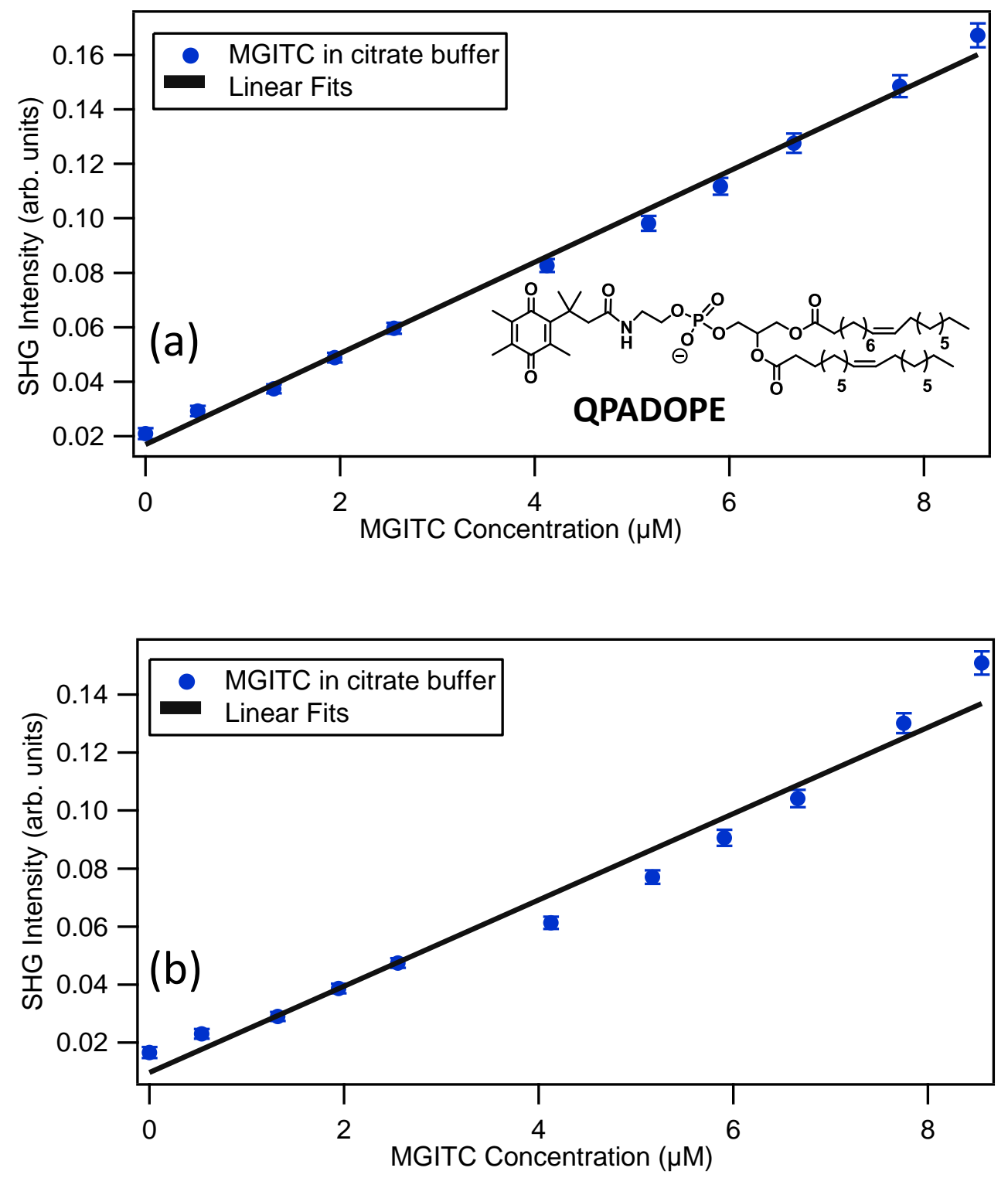

Figure S7. SHG signal intensity of (a) QPADOPE liposomes and (b) citrate buffer as a function of added MGITC concentration (blue circles) compared to linear fits (black lines). The inset of (a) shows the molecular structure of QPADOPE. 

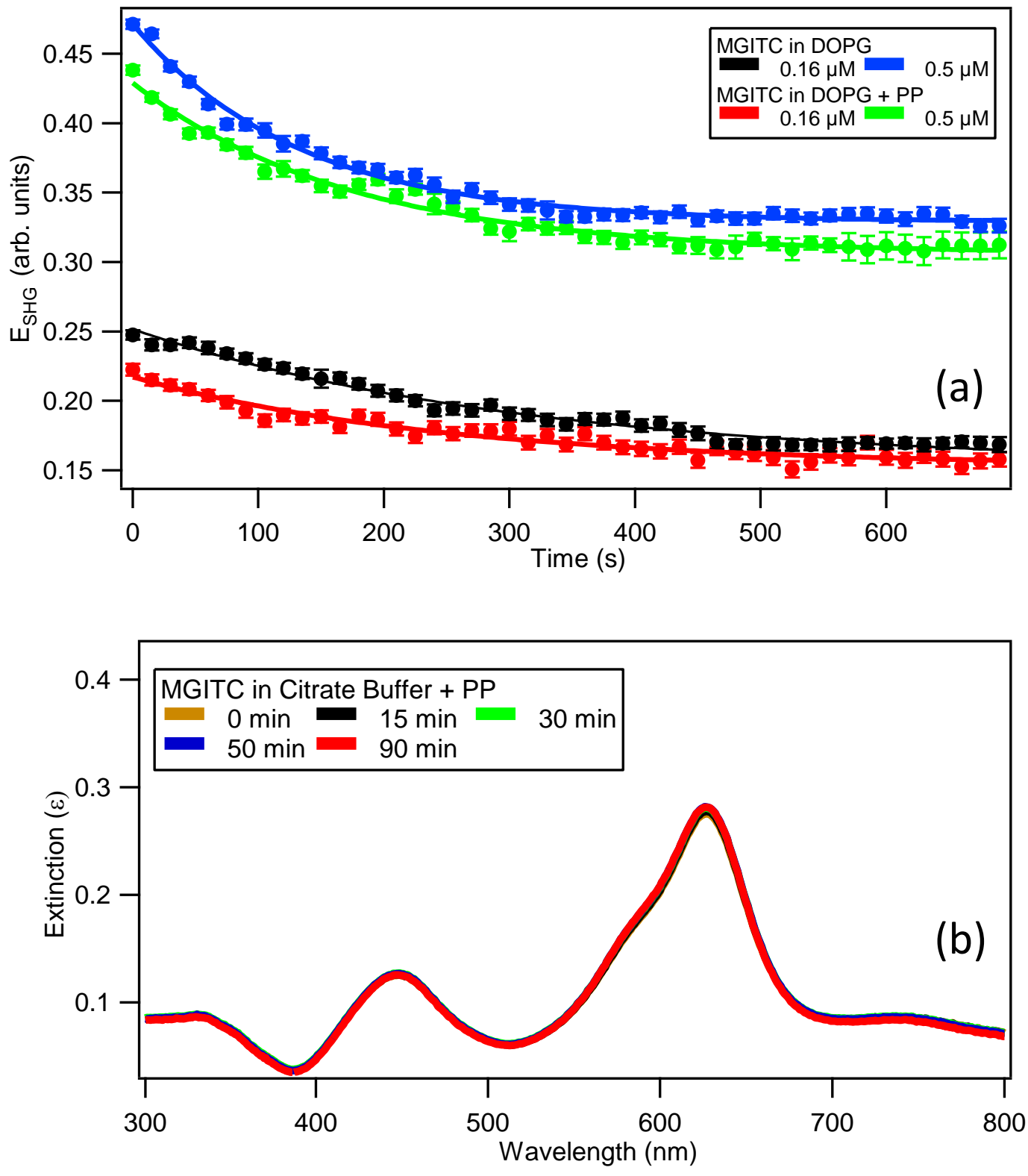

Figure S8. (a) SHG time profiles upon addition of various concentrations of MGITC to DOPG liposomes at different buffer conditions. (b) Absorption spectra of malachite green isothiocyanate in citrate buffer with $5 \mathrm{mM}$ potassium perchlorate at $\mathrm{pH} 4.0$ at various times. 


\section{Molecular Dynamics Simulations Details}

The CHARMM-GUI Membrane Builder ${ }^{6-7}$ was used to build the initial structures of DOPC and DOPG membranes. Each bilayer initially had 72 phospholipid molecules. These bilayers were then solvated with 6075 SPC/E ${ }^{8}$ water molecules using Packmol. ${ }^{9}$ Since DOPG molecules are negatively charged, $72 \mathrm{~K}^{+}$ions were added to the DOPG system to neutralize the system charge. The box dimensions of the solvated membranes were approximately $59.0 \times 62.0 \times 126.0 \AA^{3}$ for DOPC, and $57.0 \times 60.0 \times 124.0 \AA^{3}$ for DOPG. Molecular dynamics simulations were carried out using LAMMPS program (version 05 Sep 2014) ${ }^{10}$ with the all-atom general AMBER force field (GAFF). ${ }^{11}$ The partial charges of the optimized structures were calculated by the RESP fitting technique ${ }^{12-13}$ using the HF/6-31G* method in Gaussian 09 suite of programs. ${ }^{14}$ The input files for the simulations were generated using the Moltemplate ${ }^{15}$ package. The energies of these initial solvated DOPC and DOPG systems were minimized followed by a 5 ns simulation in the isothermal-isobaric (NPT) ensemble and then a $10 \mathrm{~ns}$ simulation in the canonical (NVT) ensemble. Both systems were then replicated along the $\mathrm{x}$-axis and simulated for a further $5 \mathrm{~ns}$ in the NVT ensemble. The final simulation box dimensions were approximately $95.5 \times 48.0 \times 120.0 \AA^{3}$ for DOPC systems, and $94.5 \times 50.0 \times 115.0 \AA^{3}$ for DOPG systems.

The MG and MGITC structures were built using the Avogardo ${ }^{16}$ package and the structures were optimized using Gaussian 09 suite of programs ${ }^{14}$ with the HF/6-31G* method. The partial charges were calculated by the RESP fitting technique ${ }^{12-13}$ using the HF/6-31G* method. Then four different systems were generated by inserting one molecule of either MG or MGITC with their respective counter ion $\left(\mathrm{Cl}^{-}\right.$for $\mathrm{MG}$ and $\mathrm{ClO}_{4}{ }^{-}$for $\left.\mathrm{MGITC}\right)$ into the solvated DOPC and DOPG membrane systems that were already equilibrated. Energy minimization was performed on all four systems, which were then equilibrated in the NPT ensemble for 1 ns. Each system was then 
simulated for a total of $10 \mathrm{~ns}$. The Langevin thermostat ${ }^{17}$ with a collision frequency of $1 \mathrm{ps}^{-1}$ was applied to control the temperature at $303 \mathrm{~K}$. The pressure of the system was controlled at $1 \mathrm{~atm}$ by applying the Berendsen barostat ${ }^{18}$ with a damping time of $1 \mathrm{ps}$ and an isothermal compressibility of $4.6 \times 10^{-5} \mathrm{~atm}^{-1}$. The pressure was controlled semi-isotropically (only along the z-component of the system) as has been used previously. ${ }^{19}$ The SHAKE algorithm was used to constrain the bond lengths and bond angles for the water molecules and the periodic boundary condition was used for all four systems. Electrostatic interactions were calculated using the PPPM (particleparticle particle-mesh) method ${ }^{15}$ and the non-bonded Lennard-Jones interactions were cutoff beyond $10 \AA$ A. The time step used for these simulations was $2 \mathrm{fs}$.

The umbrella sampling method ${ }^{21}$ was used to determine the free energy profile of the adsorption of the two dyes on the two membranes. The starting structures for the umbrella sampling windows for each case were taken from the respective regular canonical simulations. The collective variable was the distance in the z-direction between the center of mass (COM) of the lipid bilayer and the COM of the dye molecule. For each system 20 umbrella sampling windows were simulated with a step-size of $1.5 \AA$ and a harmonic force constant of $3 \mathrm{kcal} / \mathrm{mol}$. The simulation time for each window was $18 \mathrm{~ns}$. The weighted histogram analysis (WHAM) method $^{22-23}$ was used to derive the potential mean force for the adsorption processes of each dye molecules. Only the last $14 \mathrm{~ns}$ of each umbrella sampling window was used for this analysis. The calculation of the statistical error with the potential mean force was calculated using the block averaging method with each block having $3.5 \mathrm{~ns}$ of simulation time.

The surface of the DOPC membrane was defined as the average z-coordinates of the nitrogen atom of each DOPC molecule on one side of the membrane. Similarly, the surface of the DOPG 
membrane was determined using the average z-coordinates of the oxygen atom of each DOPG's furthest hydroxyl group on one side of the membrane.

\section{Orientation Analysis}

In order to determine the orientation of each dye molecule when they are at the surface of the DOPC and DOPG membrane, the distribution of the distance in the z-direction between different reference atoms of the dye molecule and the COM of the lipid bilayer was used. The two nitrogen atoms of the amine group and the carbon atom in the third phenyl group in each dye molecule were used as the reference atoms for this analysis (See Figure S9a and S9b). All four distributions of the distances (Figures S9c-S9f) are shown for when the dye molecules are at the surface of the membranes. Specifically, for MG and MGITC in DOPC these distances were taken from the window with the collective variable set to $22.5 \AA$. The average distance (along the z-direction), D1, between the COM of the dye molecule and the surface was found to be $0.4 \pm 0.2 \AA$ for MG in DOPC. According to Figure S9c, one of the nitrogen atoms of the amine group is directed towards the membrane and the other two atoms (nitrogen and carbon) are directed away from the DOPC membrane. The average distance (along the z-direction), D1, between the COM of the dye molecule and the surface was found to be $0.7 \pm 0.2 \AA$ for MGITC in DOPC. From Figure S9d it is clear that the carbon atom with the NCS group is directed towards the membrane. The Figures S9e and S9f show the orientations of the two dye molecules in the DOPG membrane. For the distance analyses, the window with the collective variable set to $19.5 \AA$ was used. The average distance, $\mathrm{D} 2$, between the COM of the dye molecule (defined in the previous paragraph) and the surface

was found to be $0.3 \pm 0.2 \AA$ for MG in DOPG and $0.2 \pm 0.2 \AA$ for MGITC in DOPG. In both of these systems an extra amine group nitrogen atom is also directed towards the DOPG membrane other than the group that was directed towards the DOPC membrane. 
(a)
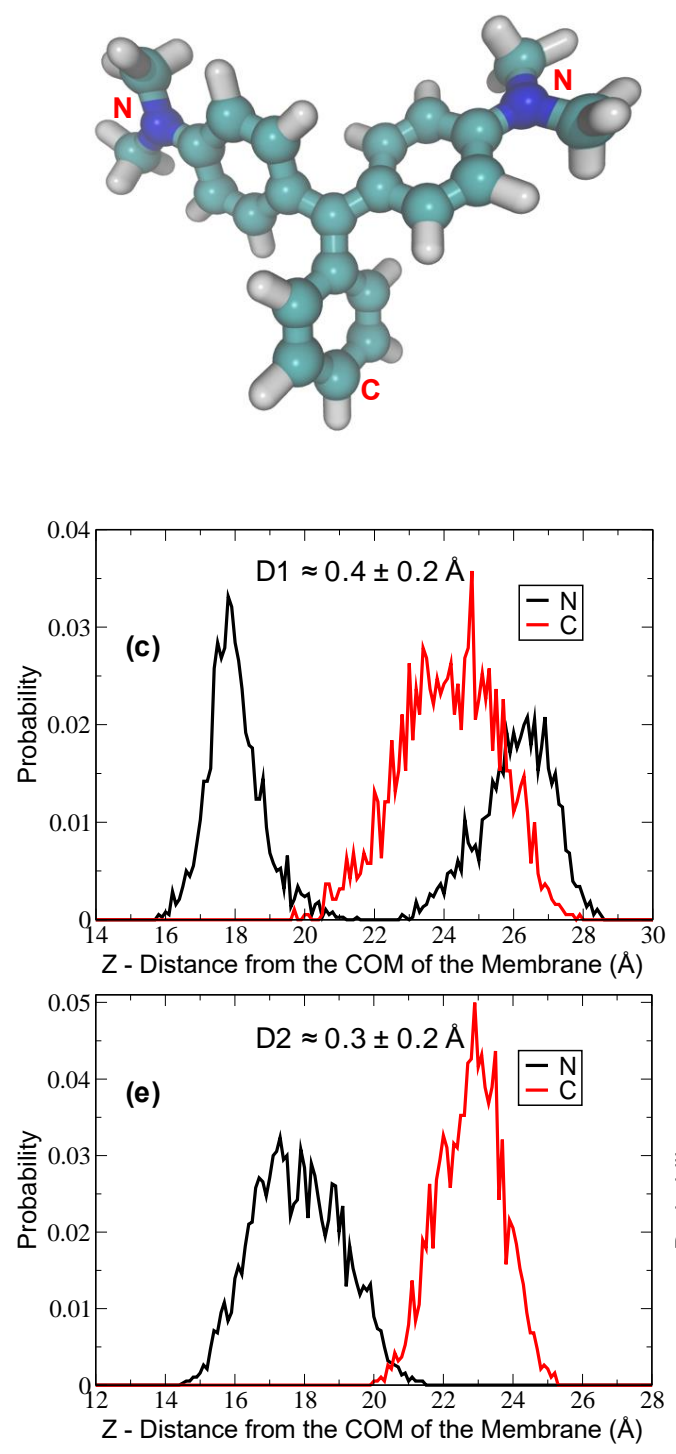

(b)
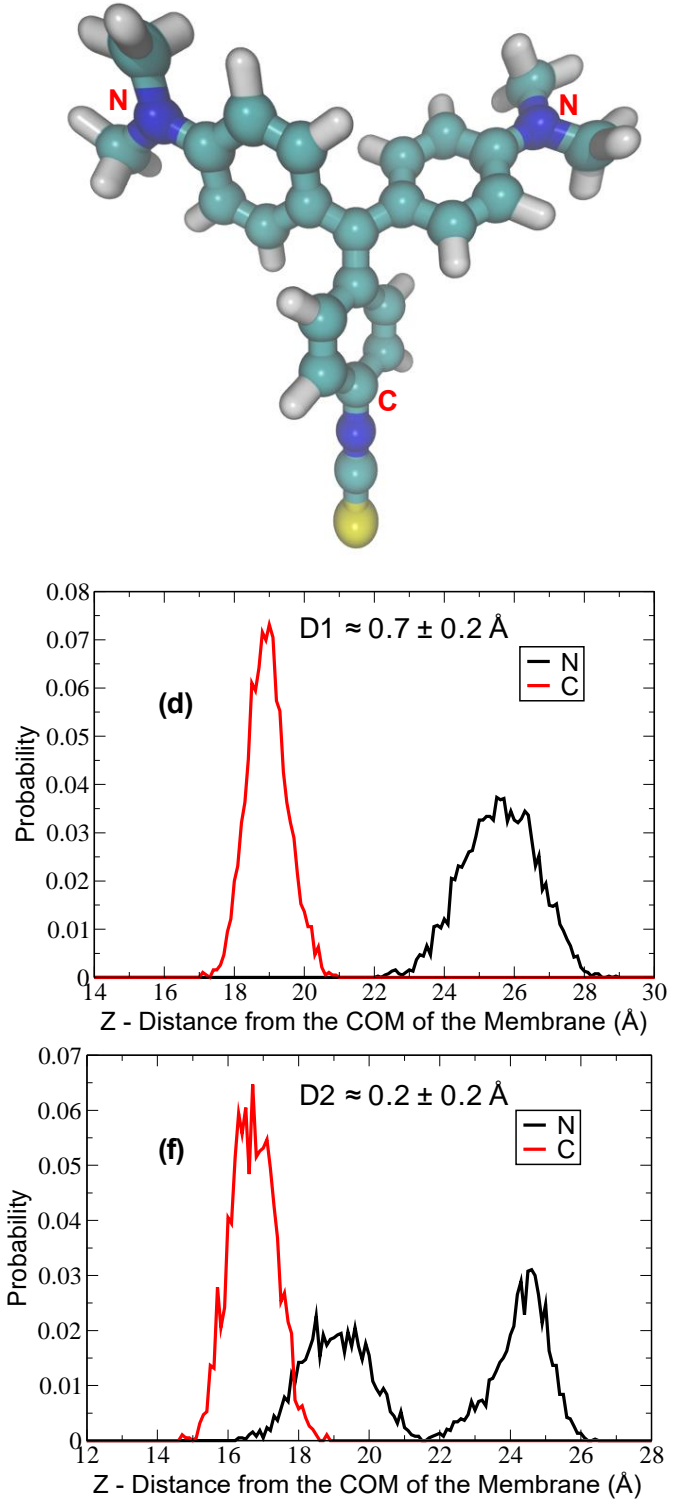

Figure S9. $\mathrm{N}$ and $\mathrm{C}$ atoms used in the distance distribution (along the z-direction) calculations for (a) MG and (b) MGITC molecules, respectively. The carbon atoms are represented in cyan, sulfur atoms in yellow, nitrogen atoms in blue, and hydrogen in white. Distributions of the distances (along the $\mathrm{z}$-direction) between the defined $\mathrm{N}$ and $\mathrm{C}$ atoms of the dye molecules in different lipid bilayer from (c) MG in DOPC, (d) MGITC in DOPC, (e) MG in DOPG, and (f) MGITC in DOPG. D1 is the distance between the COM of the dye and the surface of the DOPC membrane. D2 is the distance between the COM of the dye and the surface of the DOPG membrane. 


\section{Distribution of the Electron Density}

Using the Gaussian 09 suite of programs ${ }^{14}$ with the RESP fitting technique, ${ }^{12-13}$ the electron density distribution maps of MG and MGITC dye molecules were obtained. For these calculations, HF/6$31 G^{*}$ method and basis set were used. The two electron density maps are shown in Figure S10. Comparing the two electron density maps, the electron density is more negative on the NCS group of the MGITC molecule whereas the electron density of MG is distributed more evenly throughout the molecule, resulting in MGITC having a higher dipole moment of 10.59 Debye as compared to 2.28 Debye for MG.
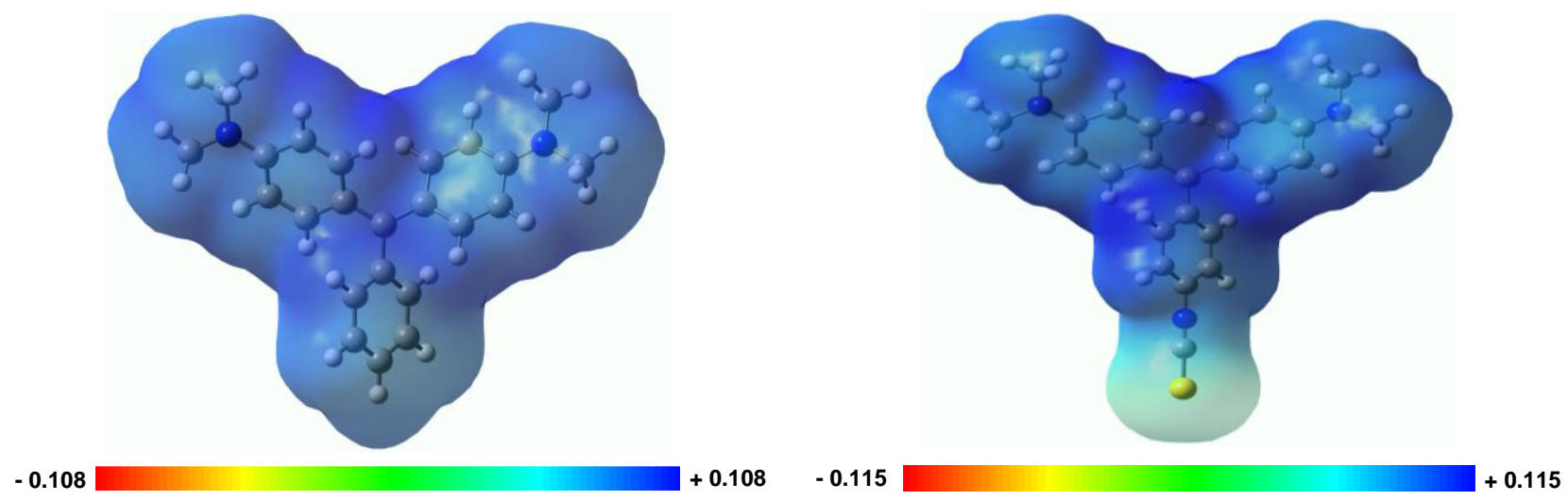

Figure S10. Distribution of the electron density around the dye molecule for (a) MG and (b) MGITC. The carbon atoms are represented in ash, sulfur atoms in yellow, nitrogen atoms in blue, and hydrogen atoms in white. 


\section{Error Analysis of Fits}

The $R^{2}$-values obtained for time-dependent exponential fits are summarized in Table S3. Similarly, the $R^{2}$-values obtained for modified Langmuir fits are shown in Table S4.

Table S3: $\boldsymbol{R}^{2}$-values of time-dependent exponential fits.

\begin{tabular}{|c|c|c|c|}
\hline & \multicolumn{3}{|c|}{$R^{2}$-Values of exponential fits } \\
\hline MGITC $(\mu \mathrm{M})$ & DOPG & DOPS & DOPC \\
\hline 0.16 & 0.93 & 0.89 & 0.89 \\
\hline 0.53 & 0.99 & 0.99 & 0.95 \\
\hline 1.06 & 0.96 & 0.98 & 0.93 \\
\hline 1.31 & 0.97 & 0.99 & 0.95 \\
\hline 1.57 & 0.97 & 0.93 & 0.97 \\
\hline 1.94 & 0.99 & 0.75 & 0.97 \\
\hline 3.44 & 0.96 & 0.97 & 0.99 \\
\hline 4.12 & 0.96 & 0.99 & 0.99 \\
\hline 4.75 & 0.99 & 0.99 & 0.99 \\
\hline
\end{tabular}

Table S4: $\boldsymbol{R}^{2}$-Values of Modified Langmuir Fits

\begin{tabular}{|c|c|c|c|}
\hline Liposome & DOPG & DOPS & DOPC \\
\hline $\mathrm{R}^{2}$-Value & 0.955 & 0.974 & 0.969 \\
\hline
\end{tabular}

\section{References}

1. Kumal, R.R.; Nguyenhuu, H.; Winter, J.E.; McCarley, R.L.; Haber H.L. Impacts of Salt, Buffer, and Lipid Nature on Molecular Adsorption and Transport in Liposomes As Observed by Second Harmonic Generation. J. Phys. Chem. C 2017, 121, 15851-15860.

2. Karam, T. E.; Haber, L. H. Molecular Adsorption and Resonance Coupling at the Colloidal Gold Nanoparticle Interface. J. Phys. Chem. C 2014, 118, 642-649.

3. Wang, H.; Yan, E.C.Y.; Liu, Y.; Eisenthal, K.B. Energetics and population of molecules at microscopic liquid and solid surfaces. J. Phys. Chem. B 1998, 102, 4446-4450.

4. Zeng, J.; Eckenrode, H. M.; Dai, H.-L.; Wilhelm, M. J. Adsorption and transport of charged vs. neutral hydrophobic molecules at the membrane of murine erythroleukemia (MEL) cells. Colloids Surf., B 2015, 127, 122-129.

5. Shang, X.; Liu, Y.; Yan, E.; Eisenthal, K. B. Effects of counterions on molecular transport across liposome bilayer: probed by second harmonic generation. J. Phys. Chem. B 2001, 105, 1281612822.

6. Jo, S.; Kim, T.; Iyer, V. G.; Im, W. CHARMM-GUI: A web-based graphical user interface for CHARMM. J. Comput. Chem. 2008, 29, 1859-65. 
7. Wu, E. L.; Cheng, X.; Jo, S.; Rui, H.; Song, K. C.; Davila-Contreras, E. M.; Qi, Y.; Lee, J.; MonjeGalvan, V.; Venable, R. M.; Klauda, J. B.; Im, W. CHARMM-GUI Membrane Builder toward realistic biological membrane simulations. J. Comput. Chem. 2014, 35, 1997-2004.

8. Berendsen, H. J. C.; Grigera, J. R.; Straatsma, T. P. The missing term in effective pair potentials. J. Phys. Chem. 1987, 91, 6269-6271.

9. Martinez, L.; Andrade, R.; Birgin, E. G.; Martinez, J. M. PACKMOL: a package for building initial configurations for molecular dynamics simulations. J. Comput. Chem. 2009, 30, 2157-64.

10. Plimpton, S. Fast parallel algorithms for short-range molecular dynamics. J. Comput. Phys. 1995, 117, 1-19.

11. Wang, J. M.; Wolf, R. M.; Caldwell, J. W.; Kollman, P. A.; Case, D. A. Development and testing of a general amber force field. J. Comput. Chem. 2004, 25, 1157-1174.

12. Bayly, C. I.; Cieplak, P.; Cornell, W. D.; Kollman, P. A. A well-behaved electrostatic potential based method using charge restraints for deriving atomic charges: the RESP model. J. Phys. Chem. 1993, 97, 10269-10280.

13. Cieplak, P.; Cornell, W. D.; Bayly, C.; Kollman, P. A. Application of the multimolecule and multiconformational RESP methodology to biopolymers: Charge derivation for DNA, RNA, and proteins. J. Comput. Chem. 1995, 16, 1357-1377.

14. M. J. Frisch, G. W. Trucks, H. B. Schlegel, G. E. Scuseria, M. A. Robb, J. R. Cheeseman, G. Scalmani, V. Barone, B. Mennucci, G. A. Petersson, H. Nakatsuji, M. Caricato, X. Li, H. P. Hratchian, A. F. Izmaylov, J. Bloino, G. Zheng, J. L. Sonnenberg, M. Hada, M. Ehara, K. Toyota, R. Fukuda, J. Hasegawa, M. Ishida, T. Nakajima, Y. Honda, O. Kitao, H. Nakai, T. Vreven, J. A. Montgomery Jr., J. E. Peralta, F. Ogliaro, M. J. Bearpark, J. Heyd, E. N. Brothers, K. N. Kudin, V. N. Staroverov, R. Kobayashi, J. Normand, K. Raghavachari, A. P. Rendell, J. C. Burant, S. S. lyengar, J. Tomasi, M. Cossi, N. Rega, N. J. Millam, M. Klene, J. E. Knox, J. B. Cross, V. Bakken, C. Adamo, J. Jaramillo, R. Gomperts, R. E. Stratmann, O. Yazyev, A. J. Austin, R. Cammi, C. Pomelli, J. W. Ochterski, R. L. Martin, K. Morokuma, V. G. Zakrzewski, G. A. Voth, P. Salvador, J. J. Dannenberg, S. Dapprich, A. D. Daniels, O“. Farkas, J. B. Foresman, J. V. Ortiz, J. Cioslowski and D. J. Fox, Gaussian 09, Revision A.02, 2009.

15. Jewett, A. I.; Zhuang, Z. Y.; Shea, J. E. Moltemplate a Coarse-Grained Model Assembly Tool. Biophys. J. 2013, 104, 169A-169A.

16. Hanwell, M. D.; Curtis, D. E.; Lonie, D. C.; Vandermeersch, T.; Zurek, E.; Hutchison, G. R. Avogadro: an advanced semantic chemical editor, visualization, and analysis platform. $J$. Cheminformatics 2012, 4, 17.

17. Schneider, T.; Stoll, E. Molecular-dynamics study of a three-dimensional one-component model for distortive phase transitions. Phys. Rev. B: Condens. Matter Mater. Phys. 1978, 17, 1302-1322.

18. Berendsen, H. J. C.; Postma, J. P. M.; van Gunsteren, W. F.; DiNola, A.; Haak, J. R. Molecular dynamics with coupling to an external bath. J. Chem. Phy. 1984, 81, 3684-3690.

19. Ding, W.; Palaiokostas, M.; Wang, W.; Orsi, M. Effects of Lipid Composition on Bilayer Membranes Quantified by All-Atom Molecular Dynamics. J. Phys. Chem. B 2015, 119, 15263-74.

20. Hockney, R. W.; Eastwood, J. W. Computer Simulation Using Particles; Adam Hilger: New York, 1989.

21. Kästner, J. Umbrella sampling. Wiley Interdiscip. Rev.: Comput. Mol. Sci. 2011, 1, 932-942.

22. Souaille, M.; Roux, B. Extension to the weighted histogram analysis method: combining umbrella sampling with free energy calculations. Comput. Phys. Commun. 2001, 135, 40-57.

23. Kumar, S.; Bouzida, D.; Swendsen, R. H.; Kollman, P. A.; Rosenberg, J. M. The weighted histogram analysis method for free-energy calculations on biomolecules. I. The method $J$. Comput. Chem. 1992, 13, 1011-1021. 